\title{
Effect of manual in-line stabilization of the cervical spine in adults on the rate of difficult orotracheal intubation by direct laryngoscopy: a randomized controlled trial \\ Effet de la stabilisation en ligne de la colonne cervicale sur l'incidence d'intubations orotrachéales difficiles par laryngoscopie directe chez l'adulte: une étude randomisée contrôlée
}

\author{
François Thiboutot, MD • Pierre C. Nicole, MD • \\ Claude A. Trépanier, MD · Alexis F. Turgeon, MD • \\ Martin R. Lessard, MD
}

Received: 12 September 2008/Revised: 4 March 2009/ Accepted: 9 March 2009/Published online: 24 April 2009

(C) Canadian Anesthesiologists' Society 2009

\begin{abstract}
Purpose Although manual in-line stabilization (MILS) is commonly used during endotracheal intubation in patients with either known or suspected cervical spine instability, the effect of MILS on orotracheal intubation is poorly documented. This study evaluated the rate of failed tracheal intubation in a fixed time interval with MILS.

Methods Two hundred elective surgical patients were randomized into two groups. In the MILS group, the patient's head was stabilized in a neutral position by grasping the patient's mastoid processes to minimize any head movement during tracheal intubation. In the control group, the patient's head rested in an optimal position for tracheal intubation. A 30-sec period was allowed to complete tracheal intubation with a \#3 Macintosh laryngoscope blade. The primary endpoint was the rate of failed tracheal
\end{abstract}

Dr. Turgeon is a Clinical Research Scholar (career awards) of the Fonds de la Recherche en Santé du Québec (FRSQ).

Presented in part at the 62nd Annual Meeting of the Canadian Anesthesiologists' Society, Toronto, ON, June 16-20, 2006.

F. Thiboutot, MD

Department of Critical Care, University of Ottawa,

Ottawa, ON, Canada

P. C. Nicole, MD - C. A. Trépanier, MD

A. F. Turgeon, MD - M. R. Lessard, MD ( $\square)$

Department of Anesthesia and Critical Care and the Centre de Recherche du CHA (Hôpital de l'Enfant-Jésus), Unité de

Traumatologie-Urgence-Soins Intensifs, Université Laval, 1401,

18e rue, Quebec City, QC G1J 1Z4, Canada

e-mail: martin.lessard@anr.ulaval.ca intubation at $30 \mathrm{sec}$. Secondary endpoints included tracheal intubation time and the Cormack \& Lehane grade of laryngoscopy.

Results Patient characteristics were similar with respect to demographic data and risk factors for difficult tracheal intubation. The rate of failed tracheal intubation at $30 \mathrm{sec}$ was 50\% (47/94) in the MILS group compared to 5.7\% (6/ $105)$ in the control group $(P<0.0001)$. Laryngoscopic grades 3 and 4 were more frequently observed in the MILS group. Mean times for successful tracheal intubation were $15.8 \pm 8.5 \mathrm{sec}$ and $8.7 \pm 4.6 \mathrm{sec}$ for the MILS and control groups, respectively (mean difference 7.1, CI $\mathrm{I}_{95 \%}$ 5.0-9.3, $P<0.0001)$. All patients who failed tracheal intubation in the MILS group were successfully intubated when MILS was removed.

Conclusion In patients with otherwise normal airways, MILS increases the tracheal intubation failure rate at $30 \mathrm{sec}$ and worsens laryngeal visualization during direct laryngoscopy.

\section{Résumé}

Objectif Bien que la stabilisation manuelle en ligne (SMEL) soit fréquemment utilisée pendant l'intubation endotrachéale chez des patients présentant une instabilité de la colonne cervicale connue ou suspectée, l'effet de la SMEL sur l'intubation orotrachéale est très peu documentée. Cette étude a évalué le taux d'échec de l'intubation trachéale dans un intervalle de temps fixe avec une SMEL. Méthode Deux cents patients devant subir des chirurgies non urgentes ont été randomisés en deux groupes. Dans le groupe SMEL, la tête du patient a été stabilisée dans une 
position neutre en saisissant les apophyses mastoïdes afin de minimiser le mouvement de la tête pendant l'intubation trachéale. Dans le groupe témoin, la tête du patient était posée dans une position idéale pour l'intubation trachéale. On a permis une période de $30 \mathrm{sec}$ pour réaliser l'intubation trachéale avec une lame de laryngoscope Macintosh no. 3. Le critère d'évaluation principal était le taux d'échec de l'intubation trachéale à 30 sec. Les critères d'évaluation secondaires étaient le temps nécessaire à l'intubation trachéale et le grade de laryngoscopie selon l'échelle de Cormack et Lehane.

Résultats Les caractéristiques des patients étaient semblables quant aux données démographiques et aux facteurs de risque d'une intubation trachéale difficile. Le taux d'échec de l'intubation trachéale à $30 \mathrm{sec}$ était de $50 \%$ (47/94) dans le groupe SMEL par rapport à 5,7\% (6/105) dans le groupe témoin $(P<0,0001)$. Des scores de Cormack et Lehane de 3 et de 4 ont été plus fréquemment observés dans le groupe SMEL. Les temps moyens pour une intubation trachéale réussie étaient de 15,8 \pm 8,5 sec et $8,7 \pm 4,6$ sec dans les groupes SMEL et témoin, respectivement (différence moyenne 7,1, IC $95 \%$ 5,0 - 9,3, $P<0,0001)$. Tous les patients chez qui l'intubation trachéale a échoué dans le groupe SMEL ont eu une intubation réussie lorsque la SMEL était abandonnée.

Conclusion Chez les patients présentant des voies aériennes normales, la SMEL augmente le taux d'échec de l'intubation trachéale à 30 sec et permet une moins bonne visualisation du larynx pendant la laryngoscopie directe.

Manual in-line stabilization (MILS) of the cervical spine is an integral part of airway management when dealing with trauma patients. ${ }^{1}$ This maneuver is performed by firmly grasping the mastoid processes of the patient thus preventing movements of the cervical spine during tracheal intubation. $^{2}$ No traction is applied on the cervical spine, but enough force to counteract the lifting force of the laryngoscopy is applied to minimize displacement of the cervical spine. While MILS is a maneuver frequently used while securing the airway of patients with a known or a potentially unstable cervical spine, its effect on the success of endotracheal intubation is poorly documented. Since MILS does limit movement of the head during tracheal intubation, it impairs visualization of the larynx, as consistently shown by a reduction in the incidence of Cormack and Lehane's grade 1 laryngeal visualization and an increased incidence of grades 2,3 and $4 .^{3-5}$ However, evaluation of laryngoscopic view with the Cormack and Lehane scale is known to have a poor inter-physician reliability, ${ }^{6}$ and it remains a surrogate marker of the difficulty of endotracheal intubation. Thus, to appropriately appreciate its impact on endotracheal intubation, the effects of MILS should be assessed directly. The objective of this study was to evaluate the effect of MILS both on the rate of failed orotracheal intubation in a fixed time interval and on the grade of laryngoscopic view in adult patients under general anesthesia. The study hypothesis was that the rate of failed tracheal intubation would be significantly greater with MILS than without.

\section{Methods}

This randomized controlled study was conducted at the Centre hospitalier affilié universitaire de Québec (Hôpital de l'Enfant-Jésus). The study was approved by the hospital ethics committee. The study population was a convenience sample of adult patients ( $\geq 18$ years of age) undergoing elective surgery under general anesthesia with a planned orotracheal intubation. Patients were evaluated on the morning of their surgery. Following an explanation of the study, the patients provided written consent. Patients were excluded based on the following criteria: refusal; surgical table precluding the application of MILS; contraindication to the medication used to induce anesthesia; impossible to ventilate by face-mask; history of difficult tracheal intubation requiring alternatives to direct laryngoscopy; anatomical anomalies of the upper airway; surgery requiring the use of a double-lumen orotracheal tube; morbid obesity (BMI $\geq 35)$; and symptomatic gastroesophageal reflux. The following preoperative risk factors for difficult tracheal intubation were recorded: modified Mallampati class, dental status, presence of retrognathism (side-view of patient reveals chin as being posterior to the plane of the face), inability to prognate, interincisal distance (or intergingival distance in toothless patients), thyromental and sternomental distances. ${ }^{7-12}$

\section{Experimental protocol}

Standard monitoring was used for all patients. Neuromuscular blockade was monitored at the adductor pollicis by supramaximal stimulation of the ulnar nerve at the wrist. End-tidal $\mathrm{CO}_{2}$ was sampled at the Y-piece of the breathing circuit. Before induction of anesthesia, the patient's head was positioned on a pillow in the sniffing position, considered as the optimal position by the anesthesiologist performing the tracheal intubation. Each of the seven participating anesthesiologists had more than 5 years of clinical experience. After pre-oxygenation by face-mask with $100 \%$ oxygen for three minutes, anesthesia was induced with sufentanil $0.2-0.5 \mu \mathrm{g} \cdot \mathrm{kg}^{-1}$, propofol $1.0-2.5 \mathrm{mg} \cdot \mathrm{kg}^{-1}$, and rocuronium $0.6-1.0 \mathrm{mg} \cdot \mathrm{kg}^{-1}$ or succinylcholine $1.5 \mathrm{mg} \cdot \mathrm{kg}^{-1}$. The patients were manually 
ventilated by face-mask until complete paralysis was achieved. At that point, the sealed pre-numbered opaque envelope, which corresponded to the study number prepared using an electronic randomization table (www.randomizer.org), was opened and the patient was allocated either to the MILS group or to the control group. In the MILS group, the pillow used to obtain the sniffing position was removed, bringing the head in a neutral position. Next, an investigator, who was crouched on the left side of the anesthesiologist performing the tracheal intubation, grasped the patient's mastoid processes and applied sufficient force to limit as much movement of the head and the cervical spine as possible during tracheal intubation. ${ }^{13}$ The same investigator (FT) applied MILS for all patients. In the control group, the patient's head remained in the sniffing position on the pillow and tracheal intubation proceeded without interference from the assistant. This study could not be conducted in a double-blind fashion since it was obviously impossible to blind the anesthesiologist to the application of MILS. In both groups, the trachea was intubated by direct laryngoscopy with a Macintosh no. 3 laryngoscope blade. The anesthesiologist was not allowed to use laryngeal pressure to optimize the laryngoscopic view or to use any instrument to facilitate tracheal intubation.

The tracheal intubation time was measured with a chronometer and was defined as the interval from introducing the laryngoscope blade into the mouth to inflating the orotracheal tube cuff. Correct positioning of the tube was confirmed by capnography. A 30 -sec period was permitted to complete endotracheal intubation. ${ }^{14-16}$ If capnography did not confirm tracheal intubation, the attempt could be resumed only if there was time remaining in this 30 -sec period. In both groups, if the patient could not be successfully intubated within $30 \mathrm{sec}$, the attempt was aborted and recorded as a failure. In the MILS group, the patient was then ventilated for $30 \mathrm{sec}$ with $100 \%$ oxygen by face-mask; the patient's head was placed in the optimal sniffing position on the pillow, and a second 30-sec tracheal intubation attempt was performed without MILS. If tracheal intubation failed again, the protocol was discontinued and the airway was managed according to the difficult tracheal intubation algorithm of the American Society of Anesthesiologists. ${ }^{17}$ In the control group, if the trachea could not be intubated within the initial 30-sec attempt, the protocol was discontinued and the airway managed as deemed appropriate by the attending anesthesiologist. If arterial saturation decreased below $90 \%$ at any time during the study, the protocol was terminated and the tracheal intubation attempt was recorded as a failure. The anesthesiologist used the Cormack and Lehane's scale to rate the grade of laryngoscopic view of the first tracheal intubation attempt. ${ }^{18}$

\section{Data analysis}

The primary endpoint was defined as the failure to intubate the trachea within the first 30-sec attempt. Sample size was calculated to identify an increase in the rate of failed tracheal intubation in the MILS group compared with the control group. In our elective surgical population, the incidence of failed orotracheal intubation by direct laryngoscopy within $30 \mathrm{sec}$ is $4 \% .{ }^{14} \mathrm{We}$ considered that an increase to $10 \%$ in the failure rate would represent a clinically significant difference. Therefore, it was determined that a sample size of 255 patients per group was required to consider Type I and Type II errors of 5 and 20\%, respectively. Four months after commencing the study, there was a shared belief among the anesthesiologists who were performing the tracheal intubations that there was a marked difference in the tracheal intubation failure rate between the two groups. For this reason, data were reviewed by one of the investigators (MRL) who was not involved in the enrolment or in the conduct of the protocol. The statistical analysis was prospectively defined and completed by intention-to-treat (in all analyses, patients were grouped according to their original group allocation) using GraphPad InStat (version 3.06 for Windows, GraphPad Software, San Diego California, USA). Continuous variables are reported as means $\pm \mathrm{SD}$. Statistical analysis was done with the Student's $t$ test for continuous data and with the Chi square test or the Fisher's exact test for proportions. All analyses were two-sided. Since the interim analysis carries an increased risk of Type I error, the level of significance was reduced to $P<0.029 .{ }^{19,20}$

\section{Results}

Following interim analysis of the data accumulated during the patients' first four months of enrolment, our reviewer (MRL) recommended that the study be terminated at that point. Of the 405 patients screened, 200 were enrolled and randomly assigned to either the MILS $(n=95)$ or the control group $(n=105)$ (Fig. 1). One patient from the MILS group was excluded immediately after randomization and before endotracheal intubation because of violation of the study protocol. The two groups were comparable for gender, age, ASA physical status, anthropometric characteristics, risk factors for difficult tracheal intubation, and medication received for anesthesia induction (Table 1).

The proportion of patients who could not be intubated within the first 30-sec attempt was much higher in the MILS group than in the control group (50.0\%, $\mathrm{CI}_{95 \%} 39.9$ 60.1 and $5.7 \%, \mathrm{CI}_{95 \%}$ 1.3-10.1, respectively; $P<0.0001$ ) (Table 2). The MILS group was also associated with a 
Fig. 1 Flow diagram of the experimental protocol. Patients of the MILS group who could not be successfully intubated within the first 30 -sec attempt had MILS removed for the second tracheal intubation attempt. MILS manual in-line stabilization

Table 1 Baseline patient characteristics and anesthetic data

Data are presented as number and percentage or mean \pm SD

MILS manual in-line

stabilization, ASA American

Society of Anesthesiologists

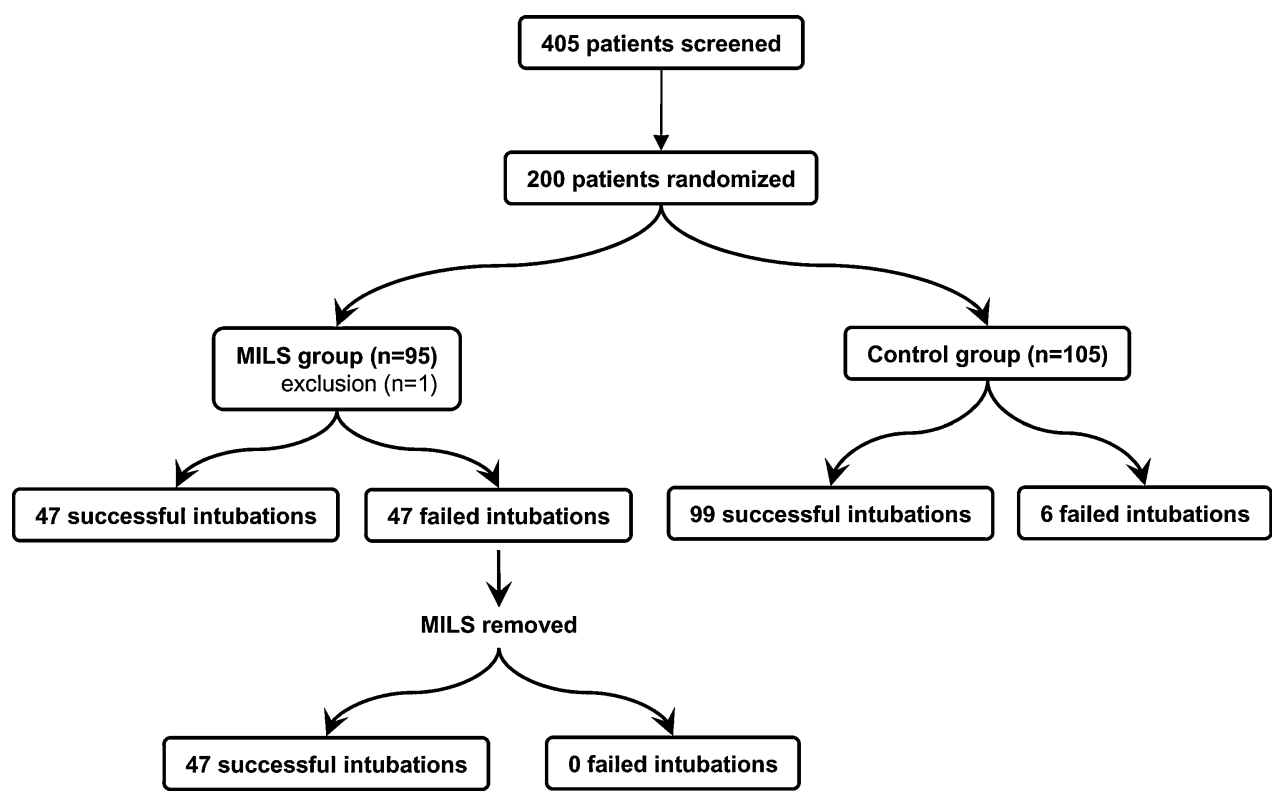

\begin{tabular}{lll}
\hline & MILS group $(n=94)$ & Control group $(n=105)$ \\
\hline Male gender & $49(52.1)$ & $49(46.7)$ \\
Age $(\mathrm{yr})$ & $40.9 \pm 14.5$ & $45.1 \pm 14.5$ \\
Weight $(\mathrm{kg})$ & $72.3 \pm 12.9$ & $70.7 \pm 14.1$ \\
Height $(\mathrm{m})$ & $1.7 \pm 0.1$ & $1.7 \pm 0.1$ \\
ASA physical status (I/II/III) & $71 / 22 / 1$ & $69 / 36 / 0$ \\
Modified Mallampati class (I/II/III/IV) & $39 / 42 / 13 / 0$ & $39 / 41 / 24 / 1$ \\
Mouth opening $<40 \mathrm{~mm}$ & $12(12.8)$ & $15(14.3)$ \\
Thyromental distance $<65 \mathrm{~mm}$ & $40(42.6)$ & $32(30.5)$ \\
Sternomental distance $<125 \mathrm{~mm}$ & $0(0.0)$ & $0(0.0)$ \\
Retrognathism & $26(27.7)$ & $26(24.8)$ \\
Inability to prognate & $1(1.1)$ & $4(3.8)$ \\
Dental status & & $62(59.0)$ \\
$\quad$ Complete dentition & $64(68.1)$ & $13(12.4)$ \\
$\quad$ Absence of dentition & $13(13.8)$ & $24(22.9)$ \\
$\quad$ Lack of all upper teeth & $15(16.0)$ & $6(5.7)$ \\
Partial lack of upper teeth & $2(2.1)$ & $0(0.0)$ \\
Other dental status & $0(0.0)$ & $188.8 \pm 60.9$ \\
Drugs for anesthesia induction & & $17.8 \pm 5.5$ \\
Propofol (mg) & $200.7 \pm 49.6$ & $40.0 \pm 17.8$ \\
Sufentanil $(\mu \mathrm{g})$ & $17.9 \pm 6.1$ & $112.7 \pm 32.2$ \\
$\quad$ Rocuronium $(\mathrm{mg})$ & $40.2 \pm 19.3$ & $15(14.3)$ \\
$\quad$ Succinylcholine $(\mathrm{mg})$ & $112.1 \pm 17.6$ & \\
Succinylcholine & $14(14.9)$ & \\
\hline & & \\
\hline
\end{tabular}

longer intubation time within successful tracheal intubations at the first attempt and with a poorer laryngoscopic view (Table 2). All 47 patients in the MILS group who could not be intubated at the first attempt were successfully intubated at the second attempt when MILS was removed.

\section{Discussion}

In this study, MILS was associated with a large increase in the rate of failed orotracheal intubation in a fixed time interval, and it also had a significant negative impact on the 
Table 2 Tracheal intubation data

\begin{tabular}{|c|c|c|c|}
\hline & MILS group $(n=94)$ & Control group $(n=105)$ & $P$ \\
\hline \multicolumn{4}{|c|}{ Failed tracheal intubation, no. (\%) } \\
\hline 1 st attempt ${ }^{\mathrm{a}}$ & $47(50.0)$ & $6(5.7)$ & \multirow[t]{2}{*}{$<0.0001$} \\
\hline 2 nd attempt ${ }^{\mathrm{b}}$ & $0(0.0)$ & - & \\
\hline \multicolumn{4}{|l|}{ Intubation time $(\mathrm{sec})^{\mathrm{c}}$} \\
\hline 1 st attempt ${ }^{\mathrm{a}}$ & $15.8 \pm 8.5$ & $8.7 \pm 4.6$ & \multirow[t]{3}{*}{$<0.0001$} \\
\hline Mean difference $\left(\mathrm{CI}_{95 \%}\right)$ & $7.1(5.0-9.3)$ & & \\
\hline 2 nd attempt ${ }^{\mathrm{b}}$ & $9.4 \pm 4.2$ & - & \\
\hline \multicolumn{4}{|l|}{ Cormack and Lehane grades ${ }^{\mathrm{d}}$} \\
\hline I & $7(7.4)$ & $77(73.3)$ & \multirow[t]{4}{*}{$<0.0001$} \\
\hline II & $32(34.0)$ & $23(21.9)$ & \\
\hline III & $44(46.8)$ & $5(4.8)$ & \\
\hline IV & $11(11.7)$ & 0 & \\
\hline
\end{tabular}

Data are presented as number and percentage or mean \pm SD

${ }^{\mathrm{a}} 1$ st attempt $=$ number of failed tracheal intubations within the first 30 -sec attempt

b 2 nd attempt $=$ number of failed tracheal intubations within the second 30-sec attempt with MILS removed, in the patients who could not be intubated at the first attempt with MILS

${ }^{\mathrm{c}}$ Intubation time $=$ time from the insertion of the laryngoscope blade to inflation of the orotracheal tube cuff during the first or the second 30sec attempt

${ }^{\mathrm{d}}$ Cormack and Lehane scale of glottic exposure = grade I, complete visualization of the vocal cords; grade II, visualization of the posterior portion of the glottis; grade III, visualization of the epiglottis only; grade IV, inability to visualize the epiglottis

time required for successful tracheal intubation and on glottic exposure during laryngoscopy. Even when MILS is performed by experienced anesthesiologists, our results show that the maneuver clearly increases the rate of difficult tracheal intubation and the risk of a failed tracheal intubation by direct laryngoscopy. Furthermore, the negative impact of MILS on tracheal intubation was confirmed by the 47 patients whose trachea could not be intubated when MILS was applied and who were successfully intubated when MILS was removed. These findings are clinically significant, since MILS is widely used in trauma patients by both anesthesiologists and emergency physicians.

It is well known that cervical spine motion is present during tracheal intubation. ${ }^{13,21,22}$ In order to reduce the risk of further spinal cord injury in patients with suspected unstable cervical spine, MILS has been proposed to immobilize the cervical spine during orotracheal intubation. Manual in-line stabilization has been shown to reduce the range of motion occurring during tracheal intubation. ${ }^{23-25}$ In contrast, other studies have shown that MILS may actually increase the range of motion of the cervical spine during direct laryngoscopy. ${ }^{26}$ However, these studies used volunteers with an intact spine or cadaver models with surgically-induced spinal lesions. No study has prospectively assessed the efficacy of MILS to prevent neurological deterioration following tracheal intubation. Evidence is limited to retrospective studies or to a few case reports describing neurological deterioration following tracheal intubation with MILS. ${ }^{27-30}$ Considering the low incidence of neurological deterioration following tracheal intubation, a huge number of patients would be required to definitively confirm the efficacy of MILS. Such a study would also be limited by obvious ethical considerations. Thus the efficacy and safety of MILS is far from established. ${ }^{31}$ Nevertheless, MILS is currently considered a standard of care and is recommended by the American College of Surgeons when intubating patients with a suspected or confirmed cervical spine injury. ${ }^{1}$

Although not designed to measure the success rate of tracheal intubation, four previous studies evaluated the effect of MILS on laryngoscopic view with a standard laryngoscope blade and yielded results in agreement with the present study. In a group of 157 patients, Nolan et al. reported a significant increase in the proportion of grades 2 and 3 laryngoscopic views when MILS was used. ${ }^{3}$ In another study, 50 patients had successive laryngoscopies, first with MILS then without the cervical stabilization maneuver. ${ }^{4}$ The incidence of grades 3 and 4 laryngoscopic views was markedly increased with the use of MILS (22\% vs $0 \%$ ). Hastings and Wood reported that the laryngoscopic view changed from grade 1 to grade 3 in $14 \%$ of their patients when MILS was applied. ${ }^{32}$ Recently, Robitaille et al. reported a 35\% incidence of grade 3 laryngoscopic view with MILS and direct laryngoscopy. ${ }^{33}$ Some direct and indirect evidences that MILS increases the rate of failed and difficult tracheal intubations have been reported by Santoni et al. ${ }^{34} \mathrm{In}$ human volunteers, they observed that the pressure applied by 
the laryngoscope blade with MILS doubled compared to control, an indirect indication that glottic view deteriorates with MILS. Moreover, such pressure may be transmitted to the cervical spine and may increase movements that MILS is trying to prevent. ${ }^{21}$ However, most of these studies used laryngoscopic view as their primary end-point. We elected to use the rate of failed tracheal intubation within a fixed time period. This method was preferred because orotracheal intubation is the final objective of direct laryngoscopy. Moreover, it is a simple objective endpoint rather than a more subjective and surrogate endpoint such as grade of laryngoscopic view, an evaluation which is known to have poor inter-physician reliability. ${ }^{6}$

The optimal duration of a tracheal intubation attempt is not well-defined. The choice of a 30-sec attempt was made a priori because it represents a reasonable duration for a tracheal intubation attempt. ${ }^{14-16}$ The Advanced Trauma Life Support (ATLS) guidelines state, "if endotracheal intubation is not accomplished within 30 seconds or in the same time required to hold your breath before exhaling, discontinue attempts...". ${ }^{35}$ Manual in-line stabilization is used in trauma patients, many of whom have associated head injury. In those patients, avoidance of hypoventilation and hypoxia is critical, making ground for limiting the length of tracheal intubation attempts. ${ }^{36}$ It may be argued that anesthesiologists would have prolonged the tracheal intubation attempts beyond $30 \mathrm{sec}$ in the clinical setting and would have successfully intubated a few more patients in the MILS group. However, considering the large clinically and statistically significant difference in failed intubations observed at $30 \mathrm{sec}$, it is unlikely that it would have reduced the incidence to a non-clinically significant difference with the control group. Thus, the results of this study should be interpreted in the clinical context of intubating a traumatized patient with a potential cervical spine injury. The actual tracheal intubation failure rate may not be as high as $50 \%$, but it is clear from this data and from other studies that MILS significantly increases the rate of difficult tracheal intubation with direct laryngoscopy. ${ }^{3,4,32-34}$

\section{Limitations}

Only 200 of the original 510 patients were included in the study. Although an interim analysis was not planned a priori, it became mandatory when the anesthesiologists participating in the study strongly believed there was a marked difference in the tracheal intubation failure rate between the two groups. The investigator who then reviewed the data had not been involved in enrolling patients or in conducting the protocol. Since an interim analysis carries an increased risk of a Type I error, the level of significance was reduced to a more conservative level of
$P<0.029 .{ }^{19,20}$ This study's results show that the rate of failed tracheal intubation using MILS was much higher than expected. Since we intended to evaluate the effect of a well-performed MILS, the MILS technique used in this study was the technique recommended most consistently, ${ }^{2,13}$ and, in each case, it was performed by the same investigator specifically trained to perform this maneuver. The investigator likely developed his expertise with the technique from experience; thus, he was better able to facilitate improved stabilization of the cervical spine and to limit movement more effectively during tracheal intubation. It may be expected that a better cervical spine immobilization would be associated with a more difficult tracheal intubation, which is different from the usual clinical setting where MILS is often applied by people with limited experience. Moreover, other techniques of cervical spine stabilization might have yielded different results. ${ }^{2}$ In the clinical setting, it is doubtful that an experienced anesthesiologist would face the challenge of intubating a patient with MILS armed only with a laryngoscope and an orotracheal tube. However, in this study, anesthesiologists were not allowed tools to facilitate tracheal intubation (e.g., stylet, gum elastic bougie) and were not allowed to apply anterior laryngeal pressure to improve the view of the vocal cords. The rate of failed tracheal intubation would likely have been lower with those aids to tracheal intubation. Since the study's objective was to measure the impact of MILS on the success of orotracheal intubation, it was decided a priori to prohibit the use of aids in order to have an unbiased evaluation of the effects of MILS on orotracheal intubation. It must also be mentioned that a Macintosh laryngoscope blade \#3 was used in our study and that the use of a straight laryngoscope blade might have yielded different results. ${ }^{21}$ Finally, we studied a population of elective surgical patients. It is likely that the influence of MILS on tracheal intubation would be even more significant when dealing with trauma patients where other factors, such as blood, oropharyngeal secretions, and tissue swelling, already impede vision during laryngoscopy. On the other hand, it has also been suggested that the assessment of tracheal intubation techniques in a simulated scenario in normal patients allows for drawing accurate conclusions that can be applied to the real clinical setting. ${ }^{37}$

\section{Conclusion}

In conclusion, our results indicate that, in an adult surgical population, MILS significantly increases the rate of failed orotracheal intubation at $30 \mathrm{sec}$, and has a significant impact on the laryngoscopic view and on the duration of the tracheal intubation. If the application of MILS is deemed indicated in a clinical situation, the physician 
should prepare accordingly and should be ready for a difficult tracheal intubation.

Acknowledgments The authors thank anesthesiologists François Caron MD FRCPC, Pierre Côté MD FRCPC, Jacques-Jules Côté MD FRCPC, Nicolas Dion MD FRCPC, and Gilles Lacroix MD FRCPC, Centre hospitalier affilié universitaire de Québec (hôpital de l'EnfantJésus), Quebec City, Quebec, Canada.

Funding This study was supported by departmental funding only.

Conflicts of interest The authors have no conflict of interest to declare.

\section{References}

1. American College of Surgeons Committee on Trauma. ATLS: Advanced Trauma Life Support Program for Doctors. 7th ed. Chicago, IL: American College of Surgeons; 2004. p. 46.

2. Gabbott DA, Baskett PJ. Management of the airway and ventilation during resuscitation. Br J Anaesth 1997; 79: 159-71.

3. Nolan JP, Wilson ME. Orotracheal intubation in patients with potential cervical spine injuries. An indication for the gum elastic bougie. Anaesthesia 1993; 48: 630-3.

4. Heath KJ. The effect on laryngoscopy of different cervical spine immobilisation techniques. Anaesthesia 1994; 49: 843-5.

5. Smith CE, Pinchak AB, Sidhu TS, Radesic BP, Pinchak AC, Hagen $J F$. Evaluation of tracheal intubation difficulty in patients with cervical spine immobilization: fiberoptic (WuScope) versus conventional laryngoscopy. Anesthesiology 1999; 91: 1253-9.

6. Ochroch EA, Hollander JE, Kush S, Shofer FS, Levitan RM. Assessment of laryngeal view: percentage of glottic opening score vs Cormack and Lehane grading. Can J Anesth 1999; 46: 987-90.

7. Samsoon GL, Young JR. Difficult tracheal intubation: a retrospective study. Anaesthesia 1987; 42: 487-90.

8. Wilson ME, Spiegelhalter D, Robertson JA, Lesser P. Predicting difficult intubation. Br J Anaesth 1988; 61: 211-6.

9. Butler PJ, Dhara SS. Prediction of difficult laryngoscopy: an assessment of thyromental distance and Mallampati predictive tests. Anaesth Intensive Care 1992; 20: 139-42.

10. Savva D. Prediction of difficult tracheal intubation. Br J Anaesth 1994; 73: 149-53.

11. El-Ganzouri AR, McCarthy RJ, Tuman KJ, Tanck EN, Ivankovich $A D$. Preoperative airway assessment: predictive value of a multivariate risk index. Anesth Analg 1996; 82: 1197-204.

12. Khan ZH, Kashfi A, Ebrahimkhani E. A comparison of the upper lip bite test (a simple new technique) with modified Mallampati classification in predicting difficulty in endotracheal intubation: a prospective blinded study. Anesth Analg 2003; 96: 595-9.

13. Crosby ET. Airway management in adults after cervical spine trauma. Anesthesiology 2006; 104: 1293-318.

14. Turgeon AF, Nicole PC, Trepanier CA, Marcoux S, Lessard MR. Cricoid pressure does not increase the rate of failed intubation by direct laryngoscopy in adults. Anesthesiology 2005; 102: 315-9.

15. Benumof $J L$, editor. Airway Management: Principles and Practice. St. Louis: Mosby; 1996. p. 143-56.

16. Ovassapian A. Fiberoptic Endoscopy and the Difficult Airway. 2nd ed. Philadelphia: Lippincott-Raven; 1996. p. 201-30.

17. American Society of Anesthesiologists Task Force on Management of the Difficult Airway. Practice guidelines for management of the difficult airway: an updated report by the American Society of Anesthesiologists Task Force on Management of the Difficult Airway. Anesthesiology 2003; 98: 1269-77.
18. Cormack RS, Lehane J. Difficult tracheal intubation in obstetrics. Anaesthesia 1984; 39: 1105-11.

19. Schulz KF, Grimes DA. Multiplicity in randomized trials II: subgroup and interim analyses. Lancet 2005; 365: 1657-61.

20. Geller NL, Pocock SJ. Interim analyses in randomized clinical trials: ramifications and guidelines for practitioners. Biometrics 1987; 43: 213-23.

21. LeGrand SA, Hindman BJ, Dexter F, Weeks JB, Todd MM. Craniocervical motion during direct laryngoscopy and orotracheal intubation with the Macintosh and Miller blades: an in vivo cinefluoroscopic study. Anesthesiology 2007; 107: 884-91.

22. Turkstra TP, Pelz DM, Shaikh AA, Craen RA. Cervical spine motion: a fluoroscopic comparison of Shikani Optical Stylet vs Macintosh laryngoscope. Can J Anesth 2007; 54: 441-7.

23. Majernick TG, Bieniek R, Houston JB, Hughes HG. Cervical spine movement during orotracheal intubation. Ann Emerg Med 1986; 15: 417-20.

24. Gerling MC, Davis DP, Hamilton RS, et al. Effects of cervical spine immobilization technique and laryngoscope blade selection on an unstable cervical spine in a cadaver model of intubation. Ann Emerg Med 2000; 36: 293-300.

25. Donaldson WF 3rd, Towers JD, Doctor A, Brand A, Donaldson $V P$. A methodology to evaluate motion of the unstable spine during intubation techniques. Spine 1993; 18: 2020-3.

26. Lennarson PJ, Smith DW, Sawin PD, Todd MM, Sato Y, Traynelis $V C$. Cervical spinal motion during intubation: efficacy of stabilization maneuvers in the setting of complete segmental instability. J Neurosurg 2001; 94(Suppl): 265-70.

27. Muckart DJ, Bhagwanjee S, van der Merwe R. Spinal cord injury as a result of endotracheal intubation in patients with undiagnosed cervical spine fractures. Anesthesiology 1997; 87: 418-20.

28. Liang BA, Cheng MA, Tempelhoff R. Efforts at intubation: cervical injury in an emergency circumstance? J Clin Anesth 1999; 11: 349-52.

29. Hastings RH, Kelley SD. Neurologic deterioration associated with airway management in a cervical spine-injured patient. Anesthesiology 1993; 78: 580-3.

30. Hastings $R H$, Marks $J D$. Airway management for trauma patients with potential cervical spine injuries. Anesth Analg 1991; 73: 471-82.

31. Manoach $S$, Paladino $L$. Manual in-line stabilization for acute airway management of suspected cervical spine injury: historical review and current questions. Ann Emerg Med 2007; 50: 236-45.

32. Hastings $R H$, Wood $P R$. Head extension and laryngeal view during laryngoscopy with cervical spine stabilization maneuvers. Anesthesiology 1994; 80: 825-31.

33. Robitaille A, Williams SR, Tremblay $M H$, Guilbert $F$, Theriault $M$, Drolet $P$. Cervical spine motion during tracheal intubation with manual in-line stabilization: direct laryngoscopy versus GlideScope videolaryngoscopy. Anesth Analg 2008; 106: 935-41.

34. Santoni BG, Hindman BJ, Puttlitz CM, et al. Manual in-line stabilization increases pressures applied by the laryngoscope blade during direct laryngoscopy and orotracheal intubation. Anesthesiology 2009; 110: 24-31.

35. American College of Surgeons Committee on Trauma. ATLS: Advanced Trauma Life Support Program for Doctors. 7th ed. Chicago, IL: American College of Surgeons; 2004. p. 56-8.

36. Brain Trauma Foundation; American Association of Neurological Surgeons; Congress of Neurological Surgeons; Joint Section on Neurotrauma and Critical Care, AANS/CNS, Bratton SL, Chestnut RM, Ghajar J, et al. Guidelines for the management of severe traumatic brain injury. I. Blood pressure and oxygenation. J Neurotrauma 2007; 24(suppl): S7-13.

37. Crosby E. Modelling the difficult airway-how real is faking it? Can J Anesth 2002; 49: 448-52. 